Publications of the Astronomical Society of the Pacific, 114:1389-1400, 2002 December

(C) 2002. The Astronomical Society of the Pacific. All rights reserved. Printed in U.S.A.

\title{
A Detailed Thermal Analysis of the Binospec Spectrograph
}

\author{
Warren R. Brown, Daniel G. Fabricant, and David A. Boyd \\ Harvard-Smithsonian Center for Astrophysics, 60 Garden Street, Cambridge, MA 02138; wbrown@cfa.harvard.edu, \\ dfabricant@cfa.harvard.edu,dboyd@cfa.harvard.edu \\ Received 2002 July 2; accepted 2002 August 15
}

\begin{abstract}
Refractive optics in astronomical instruments are potentially sensitive to temperature gradients and temperature transients. This sensitivity arises from thermally dependent refractive indices, lens spacings, and lens dimensions. In addition, thermal gradients in the instrument structure can cause undesirable image shifts at the detector that degrade instrument calibration. We have therefore undertaken a detailed thermal analysis of Binospec, a wide-field optical spectrograph under development for the converted Multiple Mirror Telescope (MMT). Our goals are to predict the temperature gradients that will be present in the Binospec optics and structure under realistic operating conditions and to determine how design choices affect these gradients. We begin our analysis by deriving thermal time constants for instrument subassemblies to estimate the magnitude of temperature gradients in the instrument and to determine where detailed thermal models are required. We then generate a low-resolution finite-difference model of the entire instrument and high-resolution models of sensitive subassemblies. This approach to thermal analysis is applicable to a variety of other instruments. We use measurements of the ambient temperature in the converted MMT's dome to model Binospec's thermal environment. In moderate conditions, the external temperature changes by up to $8^{\circ} \mathrm{C}$ over $48 \mathrm{hr}$, while in extreme conditions the external temperature changes by up to $17^{\circ} \mathrm{C}$ in $24 \mathrm{hr}$. During moderate conditions, we find that the Binospec lens groups develop $\sim 0.14^{\circ} \mathrm{C}$ axial and radial temperature gradients and that lens groups of different mass develop $\sim 0.5^{\circ} \mathrm{C}$ temperature differences; these numbers are doubled for the extreme conditions. Internal heat sources do not significantly affect these results; heat flow from the environment dominates. The instrument must be periodically opened to insert new aperture masks, but we find that the resulting temperature gradients and thermal stresses in the optics are small. Image shifts at the detector caused by thermal deflections of the Binospec optical bench structure are $\sim 0.1 \mathrm{pixel} \mathrm{hr}^{-1}$. We conclude that the proposed Binospec design has acceptable thermal properties, and we briefly discuss design changes to further reduce temperature gradients.
\end{abstract}

\section{INTRODUCTION}

The refractive optics used in modern astronomical instruments, including multiobject spectrographs and wide-field focal reducers, must typically be designed to operate over a wide range of temperatures. Refractive indices, lens spacings, and lens dimensions are all temperature-dependent, and athermal designs are necessary to compensate for changes in these quantities. However, it is difficult or impossible for the optical designer to compensate for the temperature gradients arising from temperature transients.

In this paper we study the time-dependent thermal behavior of Binospec, a wide-field, multiaperture spectrograph being developed for the $6.5 \mathrm{~m}$ converted Multiple Mirror Telescope (MMT). The mechanical layout of the Binospec spectrograph is shown in Figures 1 and 2. Binospec uses an ambitious refractive focal reducer (collimator and camera) (Fabricant, Fata, \& Epps 1998; Epps 1998) to image two adjacent $8^{\prime} \times 15^{\prime}$ fields. Binospec operates at the wide-field $f / 5$ focus of the converted MMT (Fata \& Fabricant 1993).
The collimators (see Fig. 3) each contain nine elements in three groups and produce a $200 \mathrm{~mm}$ diameter collimated beam. The cameras (see Fig. 4) each contain 10 elements in four groups that image onto a $4 \mathrm{~K} \times 4 \mathrm{~K} \mathrm{CCD}$ array. These elements are mounted in aluminum bezels with athermal, annular roomtemperature vulcanized (RTV) rubber bonds (Fata \& Fabricant 1998). The bezels have mounting bases that are attached to Binospec's optical bench.

The optics are athermalized using a new technique described in Epps \& Fabricant (2002). Briefly, the weak lenses formed in the fluid used to couple the multiplets exploit the thermally sensitive refractive index of the coupling fluid to compensate for thermally dependent lens properties: refractive indices, spacings, and dimensions. However, our concern that nonequilibrium conditions might degrade the performance of these optics motivates the present thermal analysis.

Our thermal analysis focuses on calculating the temperature gradients in the optics and the timescale of temperature changes in the instrument due to temperature transients arising from (1) external conditions in the telescope dome, (2) the inter- 


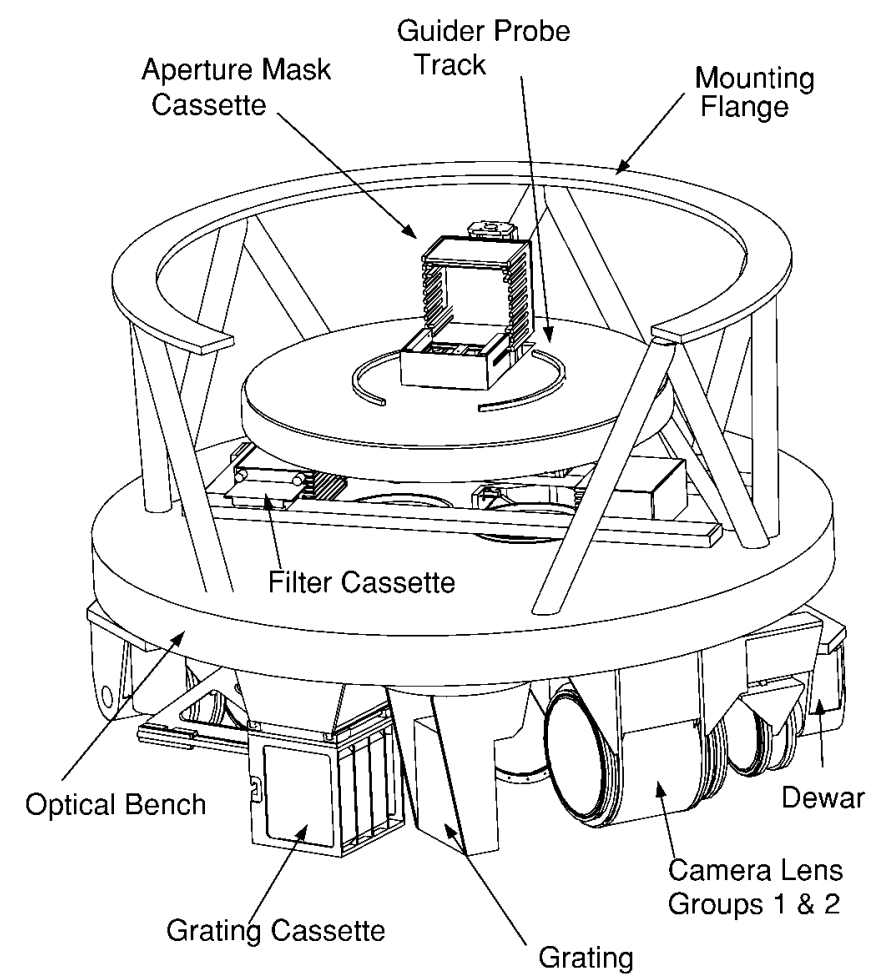

FIG. 1.-Binospec mechanical layout: top view.

mittent powering of internal heat sources (motors or other actuators), and (3) opening the instrument to change aperture masks, filters, or gratings. We also calculate the thermal stresses in the optics and the thermal deflection of the instrument optical bench. Our primary goals are to verify that Binospec will maintain its specified image quality, stability, and lateral scale under realistic operating conditions and to determine what design modifications would be beneficial.

Thermal analyses usually follow one of two general approaches. One approach is to find a closed-form analytical solution for the heat-transfer equations. Binospec is too complex for this approach. The second approach is to create a detailed thermal model of the entire instrument. We find that a complete, highly detailed model is not required to understand Binospec's thermal properties. Our approach to thermal analysis relies on a mixture of coarse and detailed models, and we believe that this approach is applicable to a variety of other instruments.

Our paper is organized to follow the steps in the analysis. We begin in $\S 2$ by laying out the basic issues of heat transfer, and in $\S 3$ we summarize the quantities needed for the heat transfer calculations. In $\S 4$, we begin the thermal analysis with calculations of thermal time constants and thermal capacitances. These analytic calculations help us to understand how quickly various Binospec components respond to temperature changes, allowing us to identify the components that dominate the heat transfer. We make detailed models of these components

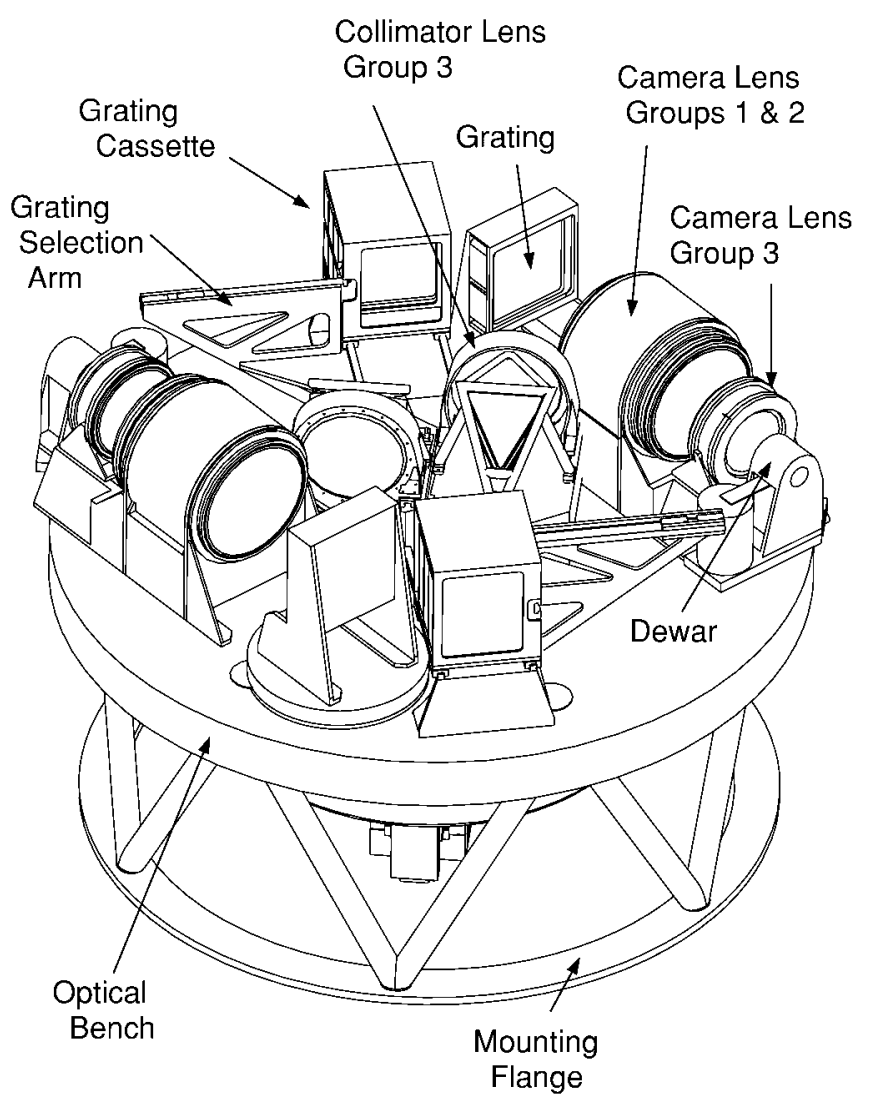

FIG. 2.-Binospec mechanical layout: bottom view. Collimator lens groups 1 and 2 are hidden by the optical bench.

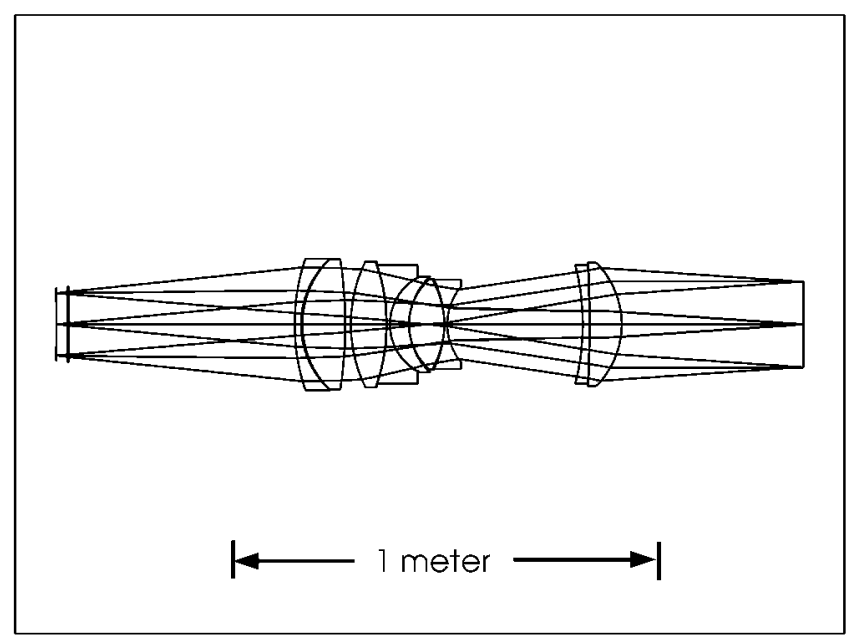

FIG. 3.-Optical layout of the Binospec collimator, with fold mirrors removed. From left to right, the optical materials are BAL15Y, S-FSL5Y, PBM2Y, PBL6Y, BAL35Y, $\mathrm{CaF}_{2}$, PBL6Y, BSM51Y, and $\mathrm{CaF}_{2}$. 


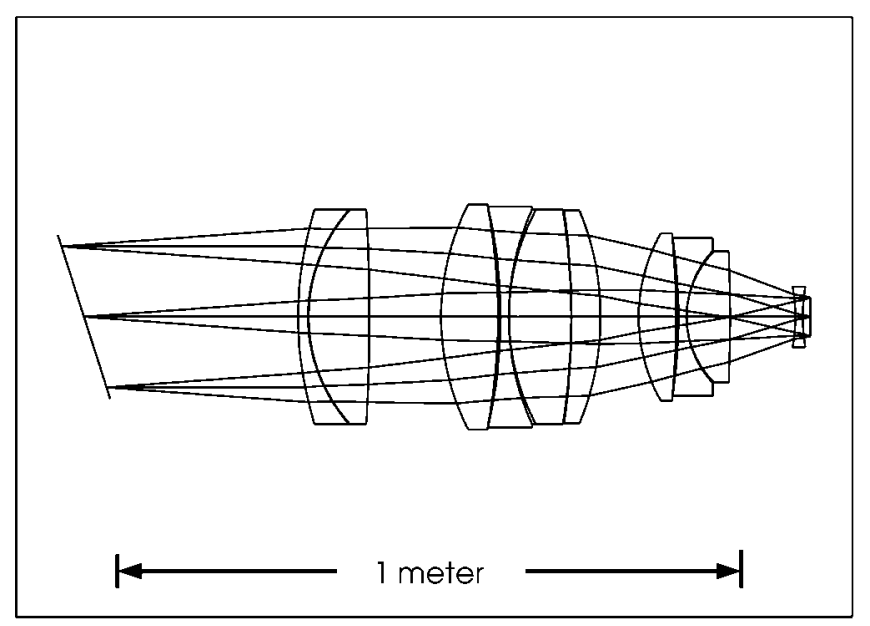

FIG. 4.-Optical layout of the Binospec camera. The last singlet serves as the Dewar window. From left to right, the optical materials are BAL35Y, $\mathrm{CaF}_{2}$, $\mathrm{CaF}_{2}, \mathrm{BAL} 35 \mathrm{Y}, \mathrm{CaF}_{2}$, PBM2Y, FPL51Y, $\mathrm{NaCl}, \mathrm{CaF}_{2}$, and BSM51Y.

and coarser models of less sensitive components. The analytic calculations also provide an important check of our final thermal models. In $\S 5$, we create thermal finite-difference models of the instrument and its subassemblies to calculate heat flows and temperature gradients. We use a low-resolution model of the entire spectrograph to provide boundary conditions for highresolution models of the subassemblies. We model in detail the thermally sensitive components, such as the collimator and camera optics, the optical bench, and the filter changer. In $\S 6$, we discuss the results from these models. We conclude in $\S 7$.

\section{HEAT TRANSFER}

Heat transfer is driven by temperature differences. There are three modes of heat transfer: conduction, convection, and radiation, all of which are important for Binospec. The heat transfer rates $Q$ for these three modes are

$$
\begin{aligned}
Q_{\text {conduction }} & =k A_{x}\left(T_{1}-T_{2}\right) / L, \\
Q_{\text {convection }} & =h A\left(T_{\text {air }}-T_{\text {surface }}\right), \\
Q_{\text {radiation }} & =\epsilon \sigma A\left(T_{\text {env }}^{4}-T_{\text {surface }}^{4}\right),
\end{aligned}
$$

where the variables are defined in Table 1.

Heat transfer is governed by the conservation of energy. In a given interval of time, the heat flow into a point plus the heat generated at that point minus the heat flow out equals the net energy gained or lost, $q_{\text {net }}$. The net energy will cause a change in temperature scaled by the thermal capacitance $m C$ :

$$
q_{\text {net }}=m C\left(T_{\text {final }}-T_{\text {initial }}\right) .
$$

\begin{tabular}{|c|c|c|}
\hline Symbol & Description & Units \\
\hline$q \ldots \ldots$ & Heat & $\mathrm{J}$ \\
\hline$Q \ldots \ldots$ & Heat transfer rate & $\mathrm{W}$ \\
\hline$\widehat{T} \ldots \ldots$ & Temperature & K \\
\hline$k \ldots \ldots$ & Conductivity & $\mathrm{W} \mathrm{m}^{-1} \mathrm{~K}^{-1}$ \\
\hline$h \ldots \ldots$ & Convection coefficient & $\mathrm{W} \mathrm{m}^{-2} \mathrm{~K}^{-1}$ \\
\hline$\sigma \ldots \ldots$ & Stefan-Boltzmann constant & $\mathrm{W} \mathrm{m}{ }^{-2} \mathrm{~K}^{-4}$ \\
\hline$L \ldots \ldots$ & Length & $\mathrm{m}$ \\
\hline$A \ldots \ldots$ & Surface area & $\mathrm{m}^{2}$ \\
\hline$A_{x} \ldots \ldots$ & Cross-sectional area & $\mathrm{m}^{2}$ \\
\hline$m \ldots \ldots$ & Mass & $\mathrm{kg}$ \\
\hline$C \ldots \ldots$ & Specific heat (at constant pressure) & $\mathrm{J} \mathrm{kg}^{-1} \mathrm{~K}^{-1}$ \\
\hline$\alpha \ldots \ldots$ & Coefficient of thermal expansion & $\mathrm{m} \mathrm{m}^{-1} \mathrm{~K}^{-1}$ \\
\hline$S \ldots \ldots$ & Stress & $\mathrm{Pa}$ \\
\hline$E \ldots \ldots$ & Young's modulus & $\mathrm{Pa}$ \\
\hline$\nu \ldots \ldots$ & Poisson's ratio & \\
\hline$\epsilon \ldots \ldots$ & Emittance & \\
\hline$F_{i j} \ldots \ldots$ & View factor & \\
\hline
\end{tabular}

Thermal finite-difference models divide a complicated system, such as the Binospec spectrograph, into a number of lump masses that are connected by the three modes of heat transfer. Discrete time steps are used to numerically solve the timedependent network of heat-transfer equations. The time derivative of the temperature is approximated by

$$
\frac{d T}{d t} \approx \frac{T(t+\Delta t)-T(t)}{\Delta t} .
$$

Temperatures are calculated at times incremented by $\Delta t$, so $t=n \Delta t$. The time step must be smaller than the shortest time constant in the model; our time steps are typically a few seconds. We refer the reader to a heat-transfer text, such as Incropera \& Dewitt (1996), for more details.

\section{PREPARATION FOR THE THERMAL ANALYSIS}

The first step in the heat-transfer analysis is to gather information on the instrument's optical and mechanical designs, the materials it uses, the environment in which it operates, and its intended operational modes. We need to determine the mass, dimensions, cross-sectional area, surface area, and surface emittance for each instrument component. The required level of detail depends on the desired resolution of the results. Temperature gradients in a shutter, for example, will usually not affect the optical performance of the spectrograph, so the shutter may be treated as a lump mass with a single cross-sectional area and surface area. Conversely, temperature gradients in the optical bench are likely to be important, so its properties should be carefully detailed. In addition, we need to determine the density, conductivity, specific heat, and coefficient of thermal expansion for each material used in the instrument. The conductivities of steel and aluminum are orders of magnitude larger than the conductivities of glass, leading to the general result that instrument structures will equilibrate faster than the optics they support. 

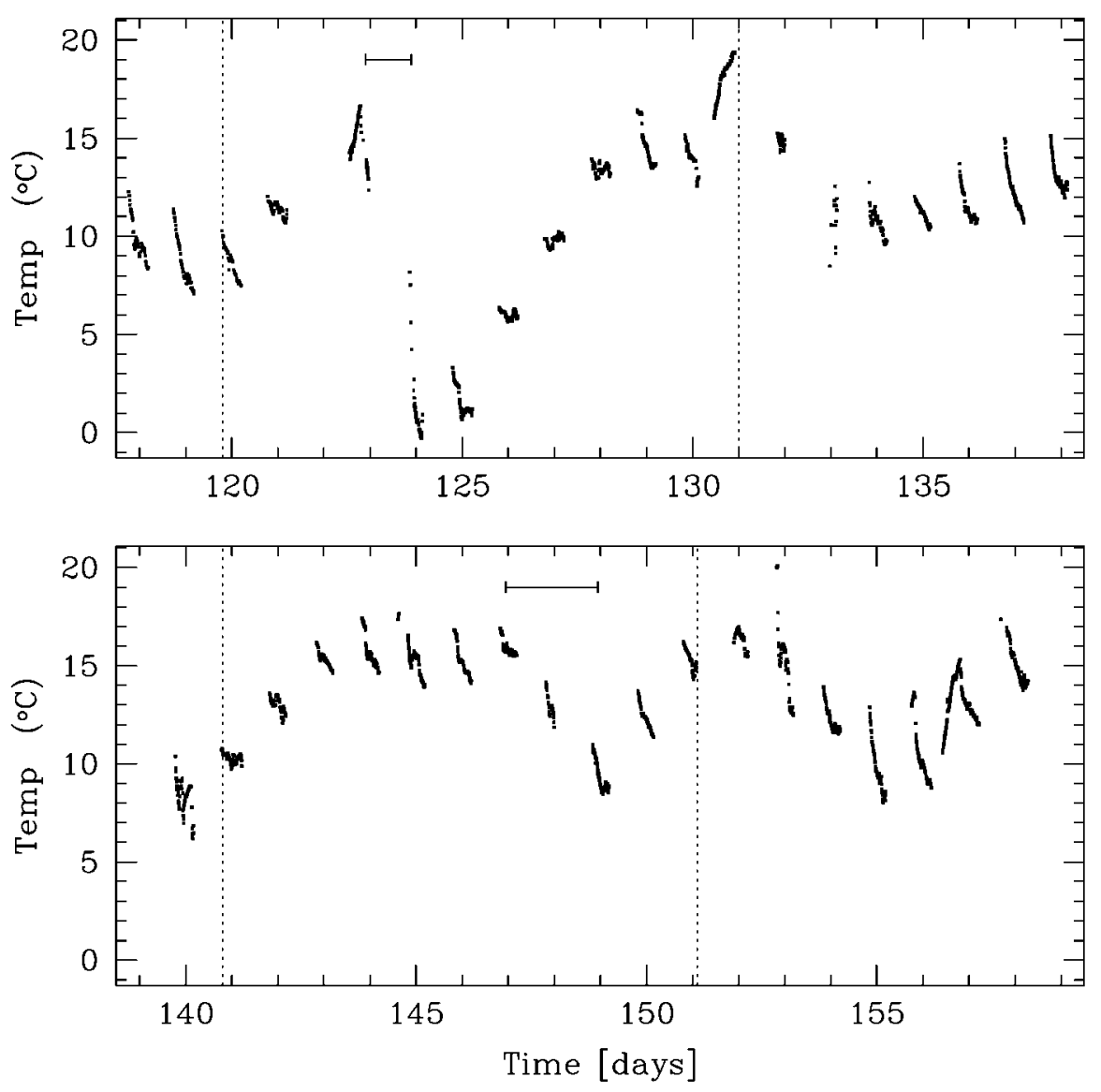

FIG. 5.-Temperatures recorded in the MMT telescope dome between 2001 April 27 and June 6 . The bar in the upper panel shows an extreme $17^{\circ} \mathrm{C}$ change in $24 \mathrm{hr}$. The bar in the lower panel shows a moderate $8^{\circ} \mathrm{C}$ change over $48 \mathrm{hr}$. The dotted lines show the time intervals we used for the extreme (upper panel) and moderate (lower panel) boundary conditions. Most of the results we describe in the text refer to the moderate temperature conditions. The temperature gradients are a factor of 2 worse for the extreme conditions.

The environment serves as the boundary condition for the thermal model, so we need to know the time dependence of the temperature in the instrument operating environment. We must also determine if the instrument's surface is directly exposed to the night sky or to a nearby heat source. In addition, the air flow speed affects the convection coefficient for the instrument's exterior surface. The amplitude and timescale of environmental temperature variations turn out to be the dominant factors in determining the heat flow and temperature gradients in Binospec.

The typical and extreme operating modes of an instrument should be identified to determine the location, power output, and duty cycle of internal heat sources. Important internal heat sources include motors, electronics boxes, and calibration lamps.

\section{FIRST-ORDER CALCULATIONS}

We begin the heat-transfer analysis with analytic calculations of thermal capacitances and time constants. We use these first- order calculations to determine the level of detail needed in our models and to check our finite-difference calculations.

\subsection{Measurements of the Thermal Environment}

Figure 5 shows MMT dome temperatures recorded between 2001 April 27 and June 6. We use the time periods between the dotted lines in the upper and lower panels to represent extreme and moderate environmental conditions, respectively. In what follows, we discuss the results appropriate for the moderate thermal environment (ambient temperature changes by up to $8^{\circ} \mathrm{C}$ over $48 \mathrm{hr}$ ). We have also examined the results for the extreme thermal environment (ambient temperature changes by up to $17^{\circ} \mathrm{C}$ in $24 \mathrm{hr}$ ); the gradients and thermal offsets almost exactly double in this case. The large temperature changes in the extreme case are usually associated with unsettled weather, so a closed dome will likely be the dominant concern. In any case, it is easy to scale the results we present for the extreme thermal environment. 
TABLE 2

Binospec Thermal Capacitances

\begin{tabular}{|c|c|c|}
\hline \multirow[b]{2}{*}{ ОВЈест } & \multicolumn{2}{|c|}{$m C\left(\mathrm{~J} \mathrm{~K}^{-1}\right)$} \\
\hline & Lens Group & Lens Barrel \\
\hline \multicolumn{3}{|c|}{ Collimator } \\
\hline Lens group $1 \ldots \ldots$ & 15700 & 5900 \\
\hline Lens group $2 \ldots \ldots$ & 26100 & 16200 \\
\hline Lens group $3 \ldots \ldots$ & 12400 & 6600 \\
\hline \multicolumn{3}{|c|}{ Camera } \\
\hline Lens group $1 \ldots \ldots$ & 20800 & 3200 \\
\hline Lens group 2 & 47000 & 7600 \\
\hline Lens group $3 \ldots \ldots$ & 13000 & 4100 \\
\hline
\end{tabular}

\subsection{Thermal Capacitances}

The thermal capacitance is the energy stored in an object per unit temperature; it is the product of the mass and the specific heat of the object $m C$. The temperature change $\Delta T$ produced by a heat input $q$ is $\Delta T=q / m C$ (see eq. [4]). The thermal capacitance is therefore a useful tool for understanding the relative thermal properties of instrument components. Table 2 lists the thermal capacitances of the Binospec lens groups and their lens barrels. We can immediately draw two conclusions. First, because the aluminum lens barrels have much smaller thermal capacitances than the lens groups, they will experience greater temperature changes than the lens groups for a given heat input. The lens barrels will therefore enforce radial temperature gradients in the lenses. We will need to model a lens group with a number of radial slices (e.g., Fig. 6). Second, the second lens groups of both the collimator and the camera have approximately twice the thermal capacitance of the neighboring lens groups and for a fixed heat input they will experience smaller temperature changes than their neighbors. We will need to model the large lens groups with a number of axial slices (see Fig. 6) to look for axial temperature gradients.

\subsection{Thermal Time Constants}

Thermal time constants are useful characterizations of instrument components. An object out of thermal equilibrium with its environment will have a temperature difference that exponentially decays to zero. We calculate the thermal time constant associated with the exponential decay, assuming that the object is isothermal and that the environment has infinite thermal capacitance. The thermal time constants for conduction, convection, and radiation are

$$
\begin{aligned}
\tau_{\text {cond }} & =m C L / k A_{x}, \\
\tau_{\text {conv }} & =m C / h A, \\
\tau_{\text {rad }} & =m C / h_{r} A,
\end{aligned}
$$

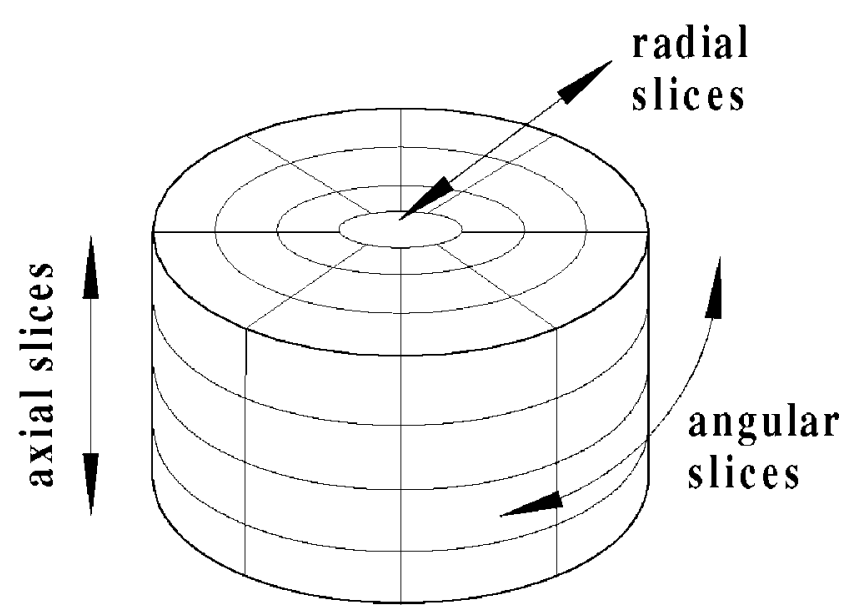

FIG. 6.-Example of a Binospec lens group model used in the detailed collimator model. The radial and axial slices are necessary to measure radial and axial temperature gradients, respectively. The angular slices are necessary to measure asymmetric diametral gradients.

where $h_{r}=\epsilon \sigma\left(T+T_{\text {env }}\right)\left(T^{2}+T_{\text {env }}^{2}\right) \simeq 4 \epsilon \sigma T_{\text {av }}^{3}$. We use $h=2$ $\mathrm{W} \mathrm{m}{ }^{-2} \mathrm{~K}^{-1}$ for still air in an enclosed cavity and $h_{r}=5 \mathrm{~W}$ $\mathrm{m}^{-2} \mathrm{~K}^{-1}$ for temperatures near $0^{\circ} \mathrm{C}$.

We model an idealized Binospec lens barrel, which is shown in Figure 7, to illustrate thermal time constant calculations. Our goal is to calculate how quickly heat conducts around a lens barrel from a motor attached to the base of the barrel. We evaluate the time constants in equations (6)-(8) for an $m=20 \mathrm{~kg}$ aluminum barrel $\left(k=164 \mathrm{~W} \mathrm{~m}^{-1} \mathrm{~K}^{-1}, C=962 \mathrm{~J} \mathrm{~kg}^{-1} \mathrm{~K}^{-1}\right)$ with radius $0.25 \mathrm{~m}$, cross-section $A_{x}=0.005 \mathrm{~m}^{2}$, and surface area $A=0.4 \mathrm{~m}^{2}$. The time constant for heat to conduct halfway around the lens barrel is $\tau_{\text {cond }}=2.6 \mathrm{hr}$.

We now consider the effects of convection and radiation on

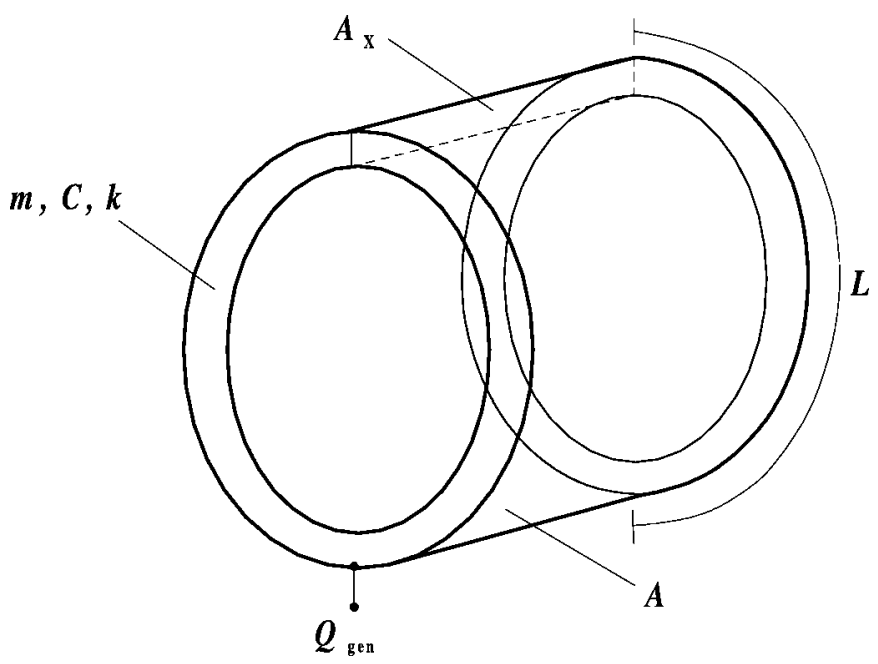

FIG. 7.-Idealized Binospec lens barrel. 

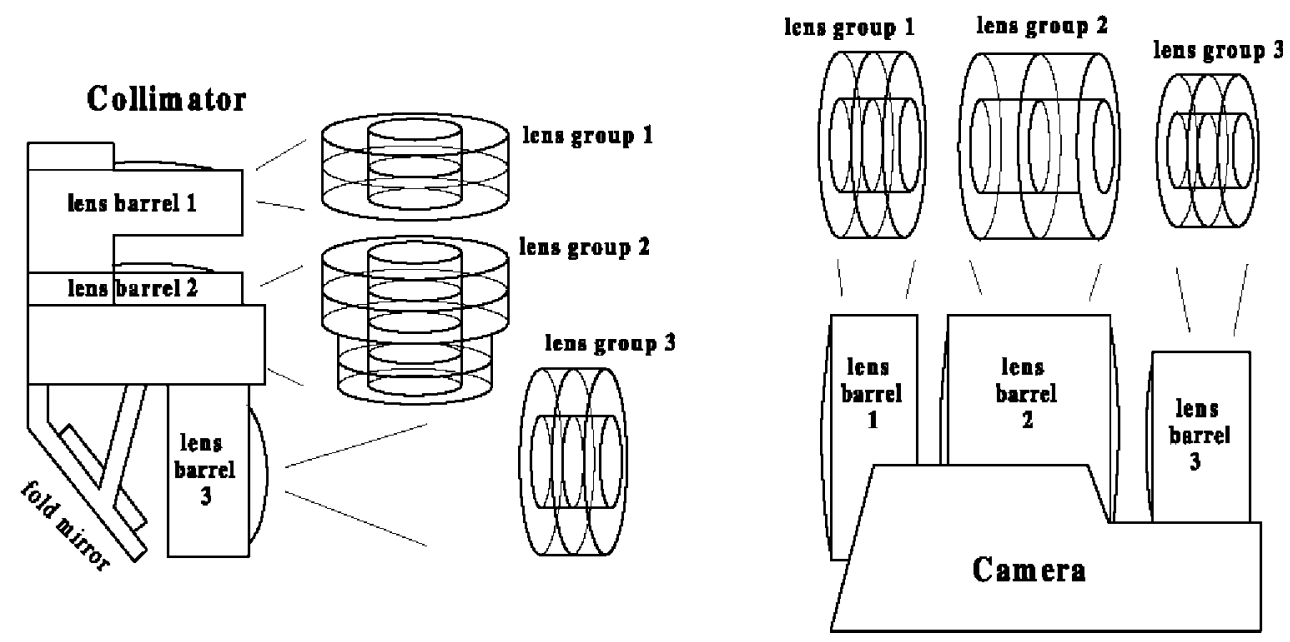

FIG. 8.-Drawings of the collimator and camera elements in the low-resolution Binospec model.

the idealized lens barrel. If its outer surface is exposed to the environment, the lens barrel will equilibrate to the environment with $\tau_{\text {conv }}=7 \mathrm{hr}$ and $\tau_{\text {rad }}=3 \mathrm{hr}$. Convective and radiative heat transfer occur in parallel, and the summed radiative and convective time constant is $2 \mathrm{hr}$.

If the interior of the lens barrel is filled with glass lenses $\left(k \simeq 0.95 \mathrm{~W} \mathrm{~m}^{-1} \mathrm{~K}^{-1}, C \simeq 650 \mathrm{~J} \mathrm{~kg}^{-1} \mathrm{~K}^{-1}\right.$ ), the time constant for heat to conduct through the surface area $A=0.4 \mathrm{~m}^{2}$ to the lens center, $L=0.25 \mathrm{~m}$, is $\tau_{\text {cond }}=18 \mathrm{hr}$. Thus, the lens barrel time constant is $\sim 10$ times shorter than the lenses it supports. The difference in time constants means that the lens barrel will act as an approximately uniform temperature boundary condition for the lenses; this allows us to use the lens barrel temperatures from our low-resolution model as boundary conditions for our high-resolution models of the lens groups. We use the detailed models, considering exactly how the Binospec lenses are attached to the lens barrels, to calculate the temperature gradients in the optics.

\section{FINITE-DIFFERENCE THERMAL MODELS}

We create finite-difference thermal models of the instrument. Each piece of the instrument, such as a lens or lens barrel, is broken into isothermal lump mass elements. Finite-difference models for a complicated system such as the Binospec spectrograph and its components require hundreds of nodes, with each node connected conductively, convectively, and radiatively to dozens of other nodes. Writing out the heat-transfer equations for such a complicated system is difficult. We use the Thermal Analysis Kit III (TAK3), a professional thermalanalysis package written by $K \& \mathrm{~K}$ Associates, to calculate the large array of heat-transfer equations. TAK3 has been previously used by engineers at the Center for Astrophysics to thermally model the Chandra X-Ray Observatory.

The heart of the TAK3 software is a general-purpose finitedifferencing thermal analyzer. The masses, dimensions, surface areas, and emittances of the elements, and the conductive, convective, and radiative pathways between them are determined by the user and typed into ASCII input files. TAK3 allows the user to easily modify the model assumptions and boundary conditions and to reanalyze the system.

\subsection{Binospec Models}

The lens barrels, mounts, and support struts are modeled with single elements in the low-resolution model of the Binospec spectrograph. Figure 8 shows that each lens group in the low-resolution model is modeled with a total of four elements, dividing the group radially and axially. This level of resolution is sufficient to estimate center-to-edge radial gradients and frontto-back axial temperature gradients in the lens groups.

Radiation exchange is relatively difficult to handle unless a complex instrument is modeled at low resolution. View factors must be calculated for every pair of elements that exchange radiation. Convection is easier to handle because each compartment in the model is assigned an air node that convectively couples the elements. We assume a convection coefficient of $2 \mathrm{~W} \mathrm{~m}^{-2} \mathrm{~K}^{-1}$ for still air inside the instrument. We use the average wind speed at the MMT, $6 \pm 3 \mathrm{~m} \mathrm{~s}^{-1}$ (Milone, Heller, $\&$ McAfee 1999), to calculate the convection coefficient exterior to the spectrograph. Sequences of temperature measurements recorded at the MMT are used as the model's boundary condition.

The high-resolution models of the collimator and camera optics, by comparison, divide each lens group into multiple axial, radial, and angular slices. Figure 9 illustrates the lens group 3 portion of the detailed camera model. Spaces between the lenses, and the RTV layers connecting the lenses to the aluminum bezels, are exaggerated for clarity. The coupling fluid that we use in the Binospec lens groups, Cargille Laser Liquid 5610 , is an excellent insulator $\left(k=0.147 \mathrm{~W} \mathrm{~m}^{-1} \mathrm{~K}^{-1}\right.$; B. Sutin 


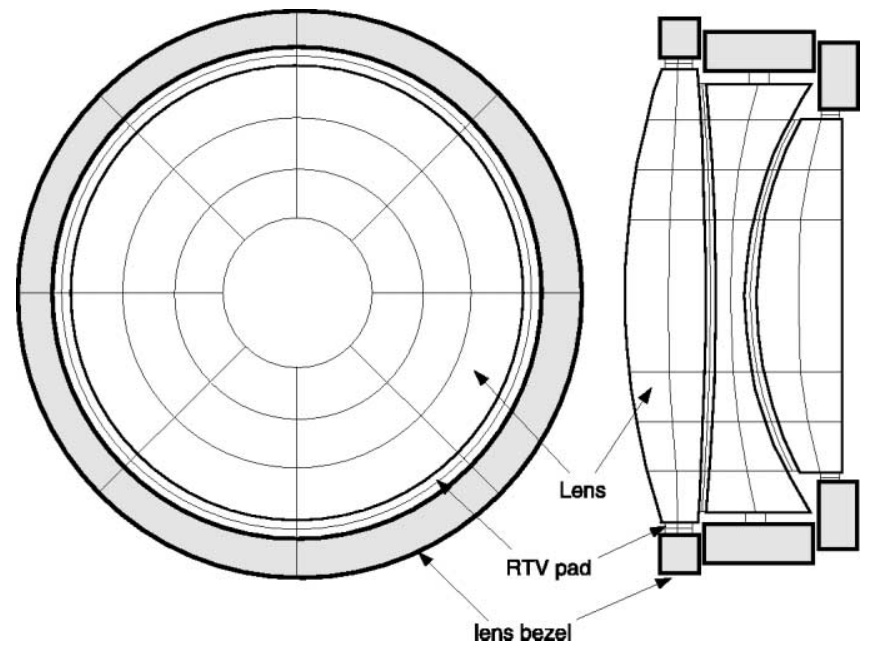

FIG. 9.-Drawing of camera lens group 3 in the detailed camera model. Each optical element is divided into multiple axial, radial, and angular slices. Spaces between the lenses and the lens bezels are exaggerated to show the coupling fluid layers and the RTV bonds.

2002, private communication), so we model the coupling-fluid layers with multiple slices as well (see Fig. 9).

We find that the axial and radial temperature gradients calculated for the lens groups generally agree to better than $0.02^{\circ} \mathrm{C}$ between the low- and high-resolution models. However, the high-resolution models reveal a new result: a diametral gradient in the lens groups because the lens barrels are mounted to the optical bench along one side. The amplitude of the diametral gradient is approximately $25 \%$ of the radial gradient.

\section{FINITE-DIFFERENCE THERMAL MODEL RESULTS}

The thermal models allow us to calculate heat flows, temperature gradients, and thermal time constants. Our basic picture of heat transfer in Binospec comes from understanding how the instrument responds to the telescope dome environment. Because we are interested in maintaining image quality in the spectrograph, our main emphasis is on the collimator and camera lens groups and the optical bench to which they are mounted. In addition, we wish to determine the thermal consequences of operating motors, as well as inserting new aperture masks, filters, and gratings. We are interested in how much insulation surrounding the spectrograph is necessary. We will use coefficients of thermal expansion to calculate thermal deformations of the instrument structure and to estimate the rate of thermally induced image drift on the detector caused by thermal deflections of the optical bench.

\subsection{Heat Flow}

Heat flow is the driving force in the thermal models. The greatest source of heat flow will dominate the thermal effects in the instrument. Because the average heat flow with the en-
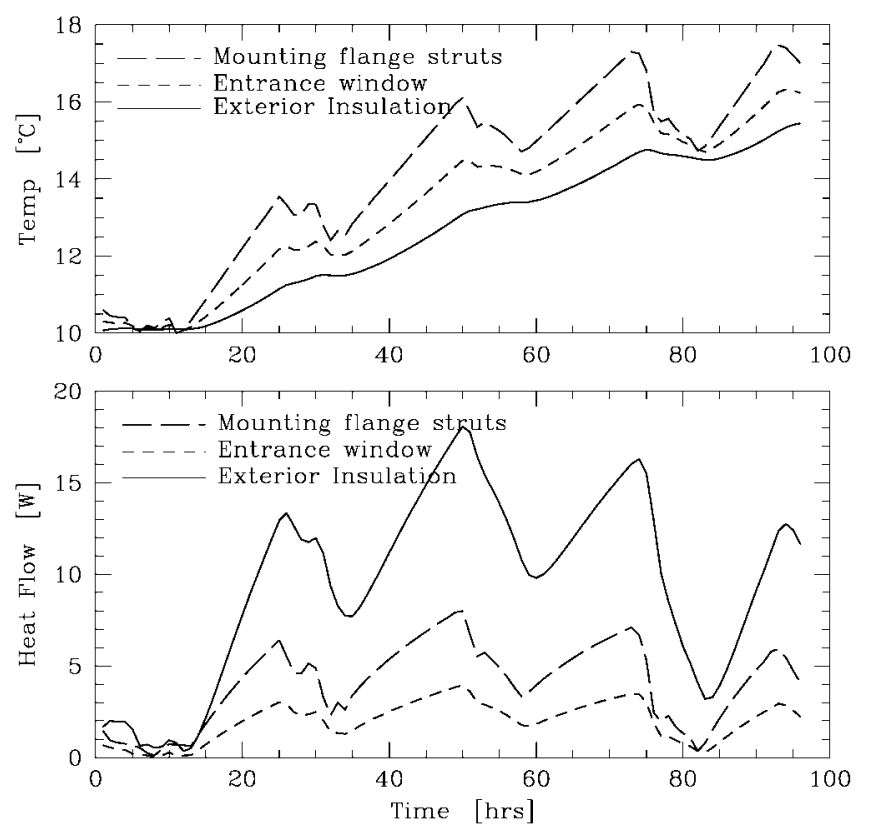

FIG. 10.-Binospec temperatures (upper panel) and heat flows (lower panel) resulting from moderate MMT temperature conditions.

vironment must be zero in the absence of internal heat sources, we consider peak heat flows. Here we compare the peak heat flow from the environment with the peak heat flow from operating motors, using the low-resolution thermal model of Binospec.

The peak heat exchange between the MMT telescope environment and Binospec is $\sim 30 \mathrm{~W}$. Approximately $60 \%$ of the heat flow passes through the large surface area of the instrument, while $40 \%$ passes through the mounting flange and the spectrograph entrance window. Figure 10 plots the temperatures and heat flows through the exterior insulation, mounting flange struts, and the entrance window for a representative $100 \mathrm{hr}$ of MMT operating conditions. This result assumes $75 \mathrm{~mm}$ thick urethane foam insulation surrounding the spectrograph and moderate MMT dome temperatures that vary by up to $8^{\circ} \mathrm{C}$ over $48 \mathrm{hr}$. Because the MMT primary mirror is actively controlled to the ambient temperature with a forced-air system, we consider the telescope mounting flange to be at ambient temperatures at all times. Table 3 summarizes the peak heat flows.

TABLE 3

Peak Heat Exchange with ENVIRONMENT

\begin{tabular}{cr}
\hline \hline Component & \multicolumn{1}{c}{$Q$} \\
$(\mathrm{~W})$
\end{tabular}



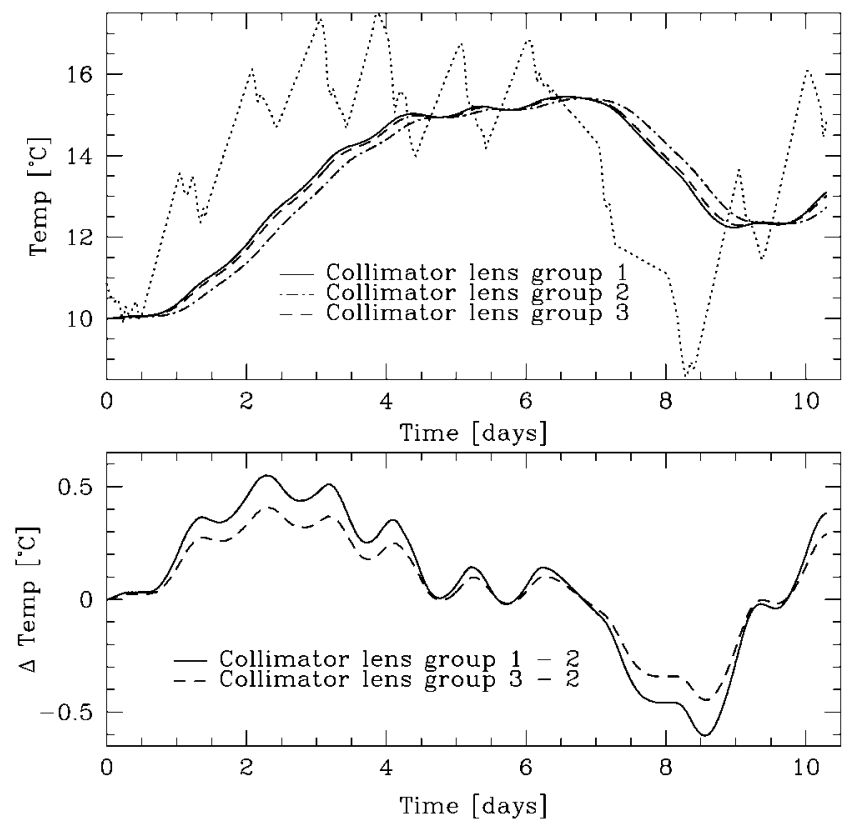

FIG. 11.-Binospec collimator lens group temperatures (upper panel) and temperature differences between lens groups (lower panel). The dotted line shows the moderate MMT dome temperatures used as the boundary condition for the model.

The Binospec optical bench is attached to the mounting flange with graphite epoxy struts (see Fig. 1). If the conductivity (and heat flow) of the graphite epoxy struts were to increase by a factor of 3 , then the time constant of the optical bench is reduced from 37 to $27 \mathrm{hr}$ and the temperature gradients in the optics are increased by $10 \%$. If the graphite epoxy struts were thermally insulated on $1 \mathrm{~cm}$ thick Delrin pads, the heat flow is reduced by a factor of 4 compared to the baseline model and the time constant of the optical bench increases from 37 to $45 \mathrm{hr}$.

The peak heat flow from internal motors when Binospec is used for spectroscopy (changing an aperture mask, rotating the gratings, and moving the guide probes every hour) or imaging (assuming a filter change every 5 minutes) is $\sim 3 \mathrm{~W}, \sim 10 \%$ of the peak heat flow from the environment. This heat-flow calculation assumes that the motors are powered only when they are required to supply torque. However, we must still look for local temperature gradients. For example, when imaging, the casings of filter motors may rise a few ${ }^{\circ} \mathrm{C}$ above the ambient temperature and induce temperature gradients in collimator lens group 1. We explore these issues in $\S 6.3$.

\subsection{Temperatures and Temperature Gradients}

One of our primary concerns is that temperature gradients may degrade the optical performance of the instrument. Conveniently, the primary output of the thermal models is tem-
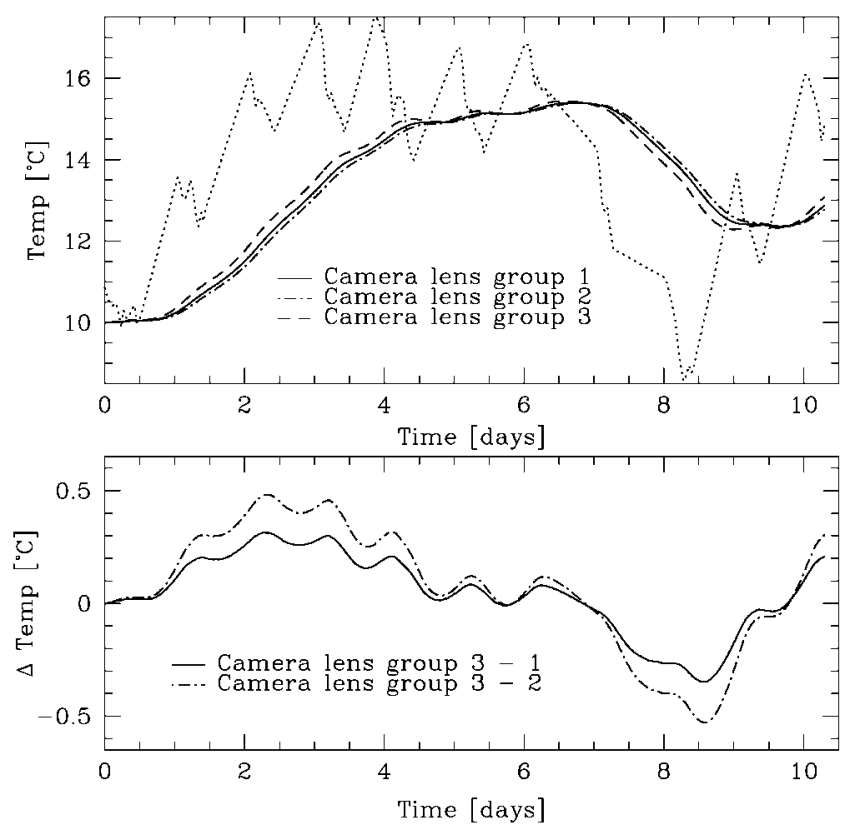

FIG. 12.-Binospec camera lens group temperatures (upper panel) and temperature differences between lens groups (lower panel). The dotted line shows the moderate MMT dome temperatures used as the boundary condition for the model.

peratures for each element as a function of time. Here we describe temperature variations in the Binospec optics.

Figure 11 illustrates the collimator lens group temperatures from the low-resolution Binospec model. The upper panel shows the cores of the three collimator lens groups responding to the moderate external temperature fluctuations. Lens group 2 has approximately twice the thermal capacitance of the other two lens groups and lags them by $6 \mathrm{hr}$; the resulting worstcase temperature differences of $\sim 0.5^{\circ} \mathrm{C}$ are shown in the lower panel. Figure 12 shows the comparable results for the camera lens groups.

Figure 13 plots the worst-case radial temperature gradient in the Binospec optics, located in collimator lens group 2. The

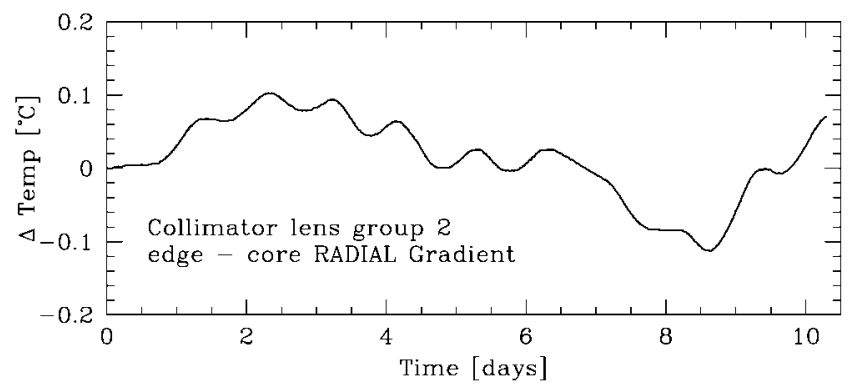

FIG. 13.-Worst-case radial temperature gradient, Binospec collimator lens group 2. 


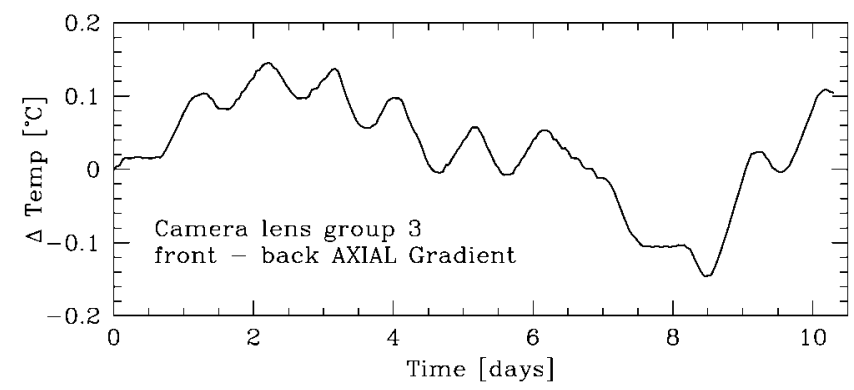

FIG. 14.-Worst-case axial temperature gradient, Binospec camera lens group 3.

detailed collimator model shows that this radial temperature gradient peaks at $0.11^{\circ} \mathrm{C}$ under moderate conditions. Similarly, Figure 14 shows the worst-case axial temperature gradient, located in camera lens group 3. The detailed camera model shows that this axial gradient peaks at $0.14^{\circ} \mathrm{C}$. Epps \& Fabricant (2002) have shown that $0.2^{\circ} \mathrm{C}$ gradients negligibly affect image quality and image scale.

We have created additional models using different environmental boundary conditions and different designs. We have compared the results with the baseline case shown in Figures 11-14. For example, we find that removing the spectrograph entrance window will result in $\sim 0.2^{\circ} \mathrm{C}(\sim 100 \%$ larger $)$ temperature gradients in the optics and a $26 \mathrm{hr}$ (30\% shorter) optical bench thermal time constant.

\subsection{Internal Heat Sources}

Internal heat sources generate heat flow that causes temperature gradients and temperature transients. Binospec uses motors to move guide probes and to change aperture masks, filters, and gratings. The motors used to change filters may be operated frequently when Binospec is used for imaging. The Binospec filter changer sits directly above collimator lens group 1 (see Fig. 1) and uses four motors, each of which may generate $16 \mathrm{~W}$ for $10 \mathrm{~s}$ during a filter change. If the filter changer is operated once every 5 minutes for $10 \mathrm{hr}$, equation (4) tells us the entire spectrograph will be heated by $\sim 0.05^{\circ} \mathrm{C}$. Although this is a small temperature change, we must consider the effect of larger local temperature gradients.

Figure 15 plots the time-dependent temperatures of a filter changer motor and the top and bottom of collimator lens group 1. The motor temperature rises $6^{\circ} \mathrm{C}$ with a time constant of $\sim 1$ hr. After a few hours of operation, the heat flow from the motors causes a $0.03^{\circ} \mathrm{C}$ top-to-bottom axial gradient in collimator lens group 1. Collimator lens group 2, on the other hand, experiences an uniform rise in temperature with no temperature gradient. We conclude that using the Binospec filter motors every 5 minutes for a few hours will cause local temperature gradients in the optics smaller than those introduced by the telescope environment (e.g., Fig. 13).
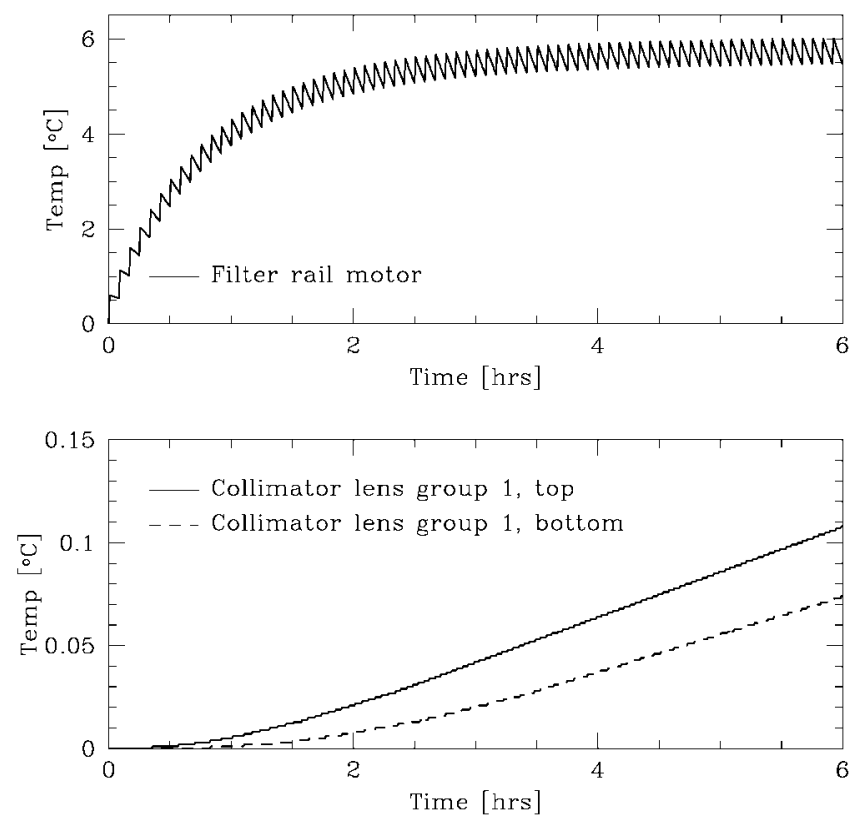

FIG. 15.-Temperature of a Binospec filter changer motor (top panel) generating $16 \mathrm{~W}$ for $10 \mathrm{~s}$ every 5 minutes, and the resulting temperature of the nearby collimator lens group 1 (lower panel).

\subsection{Time Constants}

Binospec's internal temperature gradients would be minimized if all of its internal parts could be designed to have short time constants. Unfortunately, the large thermal capacitances of the optical elements makes this impractical. The best we can do is to heavily insulate Binospec from the environment to slow heat exchange.

The thermal time constants presented here are analogous to those in equations (6)-(8). The thermal time constants quantify the response time of components in the thermal model and are calculated by starting the thermal model at an uniform temperature $10^{\circ} \mathrm{C}$ above the environment and finding the $e$-folding equilibration time for each component. Large differences between time constants of adjacent components are undesirable because they lead to large temperature gradients.

Thermal time constants for the Binospec collimator lens group 2, for example, are summarized in Table 4 . The collimator lens group 2 has an $\sim 48 \mathrm{hr}$ thermal time constant in the well-insulated Binospec design. There is a small difference in

TABLE 4

\begin{tabular}{lr}
\multicolumn{2}{c}{ Binospec Time Constants } \\
\hline \hline & $\begin{array}{c}\tau \\
\text { Collimator Lens Group } 2\end{array}$ \\
\hline Barrel $\ldots \ldots \ldots \ldots \ldots \ldots \ldots$ & 43 \\
Edge $\ldots \ldots \ldots \ldots \ldots \ldots \ldots \ldots$ & 47 \\
Core $\ldots \ldots \ldots \ldots \ldots \ldots \ldots \ldots$ & 48 \\
\hline \hline
\end{tabular}




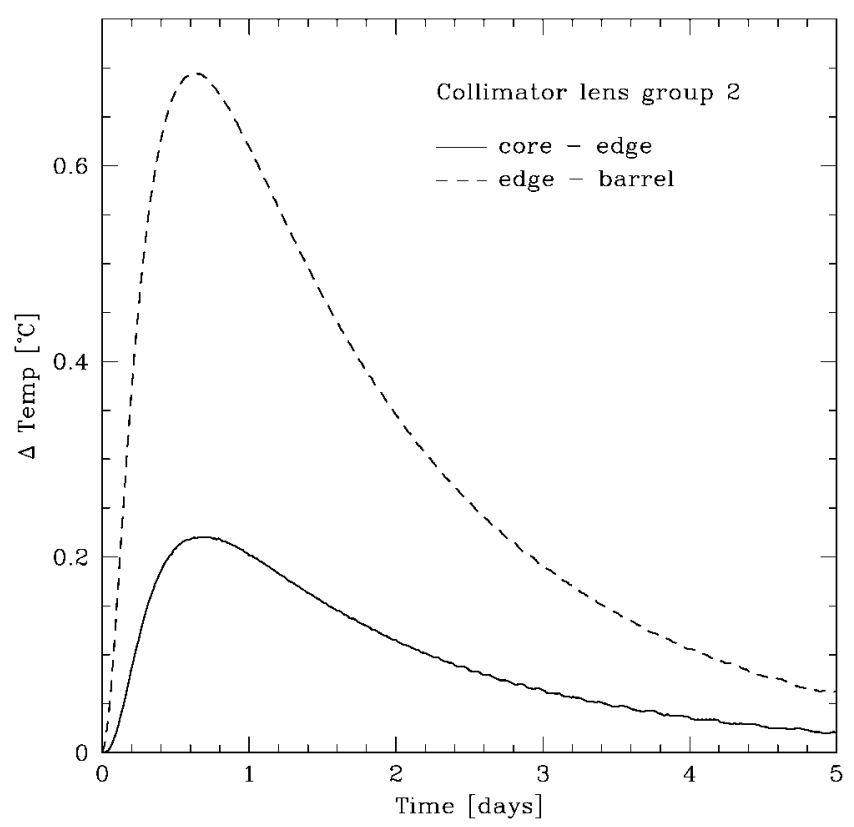

Fig. 16.-Temperature differences between the Binospec collimator lens group 2 elements, assuming that the model starts $10^{\circ} \mathrm{C}$ above the environment and then equilibrates.

time constant from the center to the edge of the collimator lens group and a corresponding $\sim 0.2^{\circ} \mathrm{C}$ temperature gradient for a $10^{\circ} \mathrm{C}$ temperature change (Fig. 16). The lens barrel, however, has a $10 \%$ shorter time constant than the lens group and a corresponding $\sim 0.7^{\circ} \mathrm{C}$ temperature gradient between the barrel and the lens group. This difference in thermal time constant is in agreement with the time constant calculations in $\S 4.3$.

\subsection{Inserting Aperture Masks and Filters}

Changing aperture masks in a multiobject spectrograph such as Binospec is a daily task. Opening the instrument to change aperture masks, filters, or gratings may expose the instrument to a troublesome thermal shock. New components placed in the instrument will most likely be at a different temperature than the well-insulated instrument and will generate heat flows and temperature gradients inside the instrument.

The aperture masks and filters are located in a compartment that includes the first collimator lens group and the top side of the optical bench (see Fig. 1). We assume that a set of 10 aperture masks of $0.5 \mathrm{~kg}$ each or a set of 12 filters of $1.2 \mathrm{~kg}$ each will be changed at a time and that the masks and filters, as well as the air introduced into the compartment, are an extreme $10^{\circ} \mathrm{C}$ hotter than the instrument. Figure 17 shows the resulting temperature changes and axial temperature gradients in the optical bench, where temperature gradients may cause significant thermal deflection. These temperature changes and gradients add to the underlying temperature changes and gradients in the baseline model.
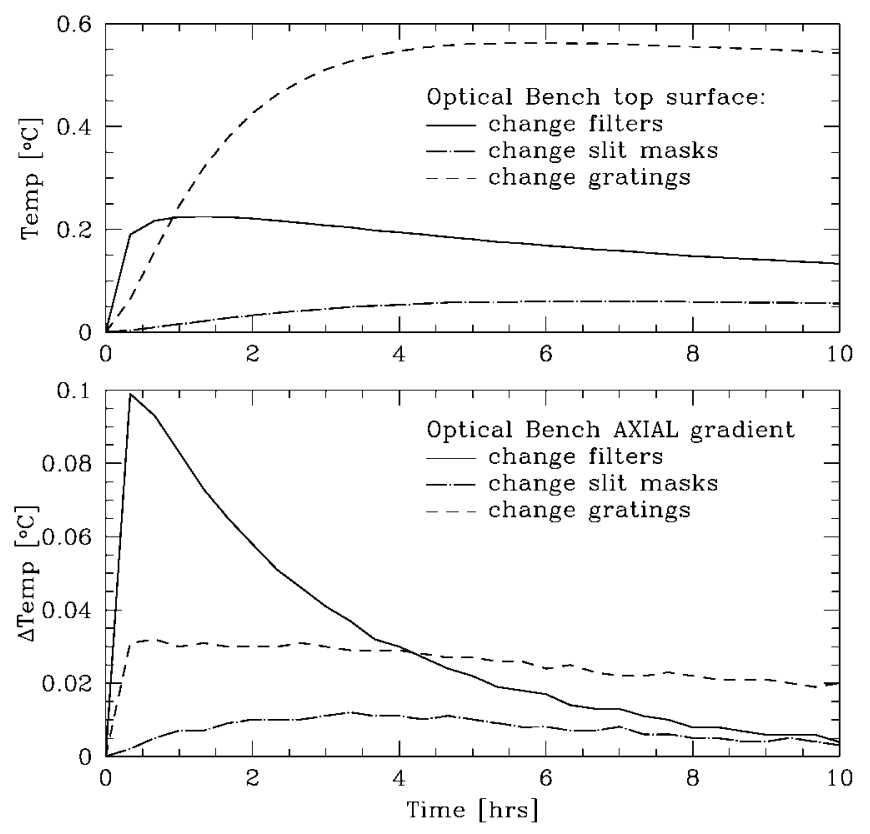

FIG. 17.-Temperature changes (upper panel) and temperature gradients (lower panel) in the optical bench resulting from inserting new filters, aperture masks, and gratings. The models assume that the new components and the surrounding air are $10^{\circ} \mathrm{C}$ above the ambient instrument temperature. These temperature changes and gradients add to the underlying temperature changes and gradients in the baseline model.

For the case of inserting new aperture masks, the optical bench is heated $0.05^{\circ} \mathrm{C}$ and experiences a $0.01^{\circ} \mathrm{C}$ axial gradient with a time constant of $3 \mathrm{hr}$. For the case of inserting new filters, the optical bench is heated $0.1^{\circ} \mathrm{C}$ and experiences a peak $0.1^{\circ} \mathrm{C}$ axial gradient with a time constant of $4 \mathrm{hr}$. The first collimator lens group, on the other hand, experiences a nearly uniform $\sim 0.1^{\circ} \mathrm{C}$ rise with a time constant of $4 \mathrm{hr}$. These temperature changes scale with the initial $10^{\circ} \mathrm{C}$ temperature difference. We conclude that the temperature gradients induced by inserting aperture masks or filters are small and will equilibrate with an acceptable time constant.

The six diffraction gratings have 10 times the thermal capacitance of the aperture masks and filters, and so changing gratings is more of an issue. The six $9 \mathrm{~kg}$ gratings plus their $7 \mathrm{~kg}$ grating holders will heat the entire instrument by $0.5^{\circ} \mathrm{C}$. Secondly, the gratings have a poor conduction path to the rest of the instrument, and their equilibration time constant is long. Finally, the gratings directly view the collimator and camera lens groups. For an initial $10^{\circ} \mathrm{C}$ temperature difference, the gratings will induce a $0.1^{\circ} \mathrm{C}$ axial gradient through collimator lens group 3 and camera lens group 1.

\subsection{Thermal Stress}

Temperature gradients cause thermal stress in the optical elements. In this section, we estimate the thermal stresses in a simplified Binospec lens. We approximate a lens by a cylin- 
drical disk and use the following stress equations from Roark \& Young (1989). The total stress caused by an uniform axial temperature gradient $\Delta T_{z}$ in a disk clamped around its edge is given by

$$
S=\frac{1}{2} \alpha E \Delta T_{z} /(1-\nu),
$$

where $S$ is the stress, $\alpha$ is the coefficient of thermal expansion, $E$ is the Young's modulus, and $\nu$ is the Poisson's ratio of the material. The thermal stress caused by a $0.1{ }^{\circ} \mathrm{C}$ axial gradient in a typical glass lens $\left(\alpha \simeq 8 \times 10^{-6{ }^{\circ}} \mathrm{C}^{-1}, E \simeq 70 \times 10^{9} \mathrm{~Pa}\right.$, $\nu \simeq 0.24)$ is 5 psi. The thermal stress caused by a $0.1^{\circ} \mathrm{C}$ axial

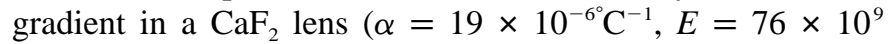
$\mathrm{Pa}, \nu=0.26)$ is 14 psi.

The stress in a disk caused by an uniform radial temperature gradient $\Delta T_{r}$ is given by

$$
\begin{aligned}
& S_{r}=\frac{1}{3} \alpha E \Delta T_{r}\left(r_{o}-r\right) / r_{o}, \\
& S_{t}=\frac{1}{3} \alpha E \Delta T_{r}\left(r_{o}-2 r\right) / r_{o},
\end{aligned}
$$

where $S_{r}$ and $S_{t}$ are the stresses in the radial and tangential directions, respectively, and $r_{o}$ is the outer radius. Note that both the radial and tangential stresses peak in the lens center $(r=0)$. If we sum the radial and tangential stress in the lens center, the total stress caused by a $0.1^{\circ} \mathrm{C}$ radial gradient is 5 psi for a typical glass lens and 14 psi for $\mathrm{CaF}_{2}$. These stress levels are insignificant.

\subsection{Thermal Deflections}

One of our design goals is to minimize image drift at the detector. Image drift degrades image quality and the wavelength calibration during a long spectroscopic exposure. The thermal stability of the Binospec optical bench is a key issue in understanding image drifts. We can simply estimate the thermal deflection of the Binospec optical bench by modeling the bench as an unconstrained circular disk. The disk geometry is a good description of the Binospec optical bench, and the formulae can be taken directly from Roark \& Young (1989). The radius of curvature $R$ of a disk with a uniform, top-to-bottom temperature difference $\Delta T_{z}$ is

$$
R=\frac{t}{\alpha \Delta T_{z}},
$$

where $\alpha$ is the coefficient of thermal expansion and $t$ is the thickness of the disk. The thermal deflection $\theta$ caused by the axial temperature difference is

$$
\tan \theta=\frac{r / R}{\sqrt{1-(r / R)^{2}}} .
$$

Binospec has a cylindrical aluminum optical bench that is $2.14 \mathrm{~m}$ in diameter and $0.15 \mathrm{~m}$ thick. In our thermal models, the optical bench experiences up to $\pm 0.1^{\circ} \mathrm{C}$ axial temperature gradients. The resulting tilt at the edge of the optical bench is \pm 3 ".5. We calculate the same tilt when we make a detailed finite-element model of the optical bench. The optical bench responds to external temperatures that vary with a timescale of $12 \mathrm{hr}$, so the rate of deflection at the edge of the optical bench is $3.5 / 12=0.3 \mathrm{hr}^{-1}$. If we insert new filters into Binospec, the time constant is $4 \mathrm{hr}$ and the rate of deflection at the edge of the optical bench is $0.9 \mathrm{hr}^{-1}$.

We use the ZEMAX optical design software (published by Focus Software, Inc.) to estimate image shifts at the detector due to deflection of various optical elements mounted on the optical bench. The grating is by far the most sensitive optical element: a 4 " deflection of the grating leads to a 1 pixel shift on the detector. The grating experiences $\frac{2}{3}$ of the total optical bench deflection, so the image drift due to external temperature changes is an acceptable 0.05 pixel $^{\mathrm{hr}^{-1}}$. We conclude that thermal deflection of the optical bench by external temperature changes will not significantly degrade an hour-long spectroscopic observation. The corresponding result for image drifts following the insertion of new filters is $0.15 \mathrm{pixel} \mathrm{hr}^{-1}$, so we will want to minimize filter changes.

More extreme temperature gradients may be created by internal heat sources, such as a hot motor mounted on the optical bench. We turn to mechanical finite-element modeling to assess the deflection caused by a hot spot on the optical bench. We find that a $0.1^{\circ} \mathrm{C}$ hot spot, with a time constant of $1.5 \mathrm{hr}$, will cause a local 0.2 deflection of the Binospec optical bench. A $16 \mathrm{~W}$ motor mounted to the optical bench on $6 \mathrm{~mm}$ thick Delrin standoffs will produce a $0.1{ }^{\circ} \mathrm{C}$ hot spot if powered with a $3 \%$ duty cycle. Figure 18 shows the deflection as a function of distance from the spot. If the grating mount were located next to this hot spot, the resulting image drift rate would be 0.03 pixel $\mathrm{hr}^{-1}$. This is similar to the drift rate caused by the $0.1^{\circ} \mathrm{C}$ gradient across the optical bench from external temperature changes and suggests that we want to be careful how we mount motors near the grating. Thermal standoffs are effective in reducing the magnitude of hot spots.

\section{CONCLUSIONS}

We have presented the thermal-analysis techniques that we have used to study the thermal behavior of Binospec, a widefield, multiobject optical spectrograph that uses high-performance refractive optics. We begin our analysis by calculating thermal time constants and thermal capacitances to understand the scale of temperature variations in the instrument and 


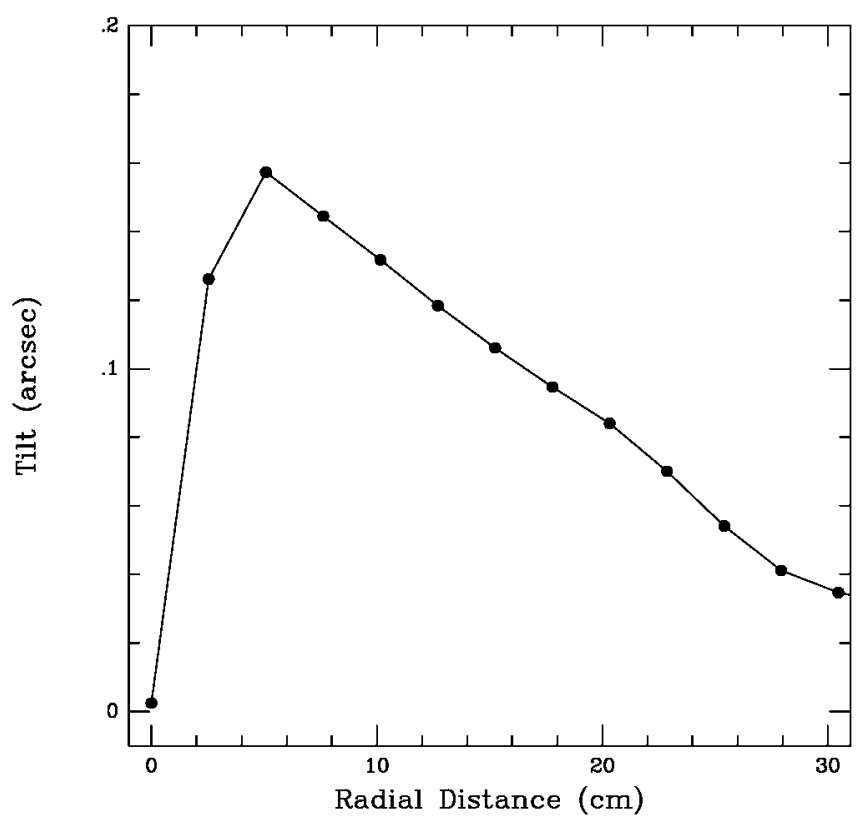

FIG. 18.-Deflection caused by a $0.1^{\circ} \mathrm{C}$ hot spot at the center of the Binospec optical bench.

to determine what areas of the instrument require detailed modeling. We then generate a low-resolution finite-difference thermal model of the entire spectrograph and high-resolution models of the collimator and camera optics, optical bench, and filter changer. We use temperature data recorded at the MMT to set the boundary conditions for the models. Conduction, convection, and radiation are all important modes of heat transfer in the instrument.

In normal operation, we find that the Binospec lens groups will experience up to $\sim 0.14^{\circ} \mathrm{C}$ axial and radial temperature gradients and that lens groups of different mass will have temperatures that differ by $\sim 0.5^{\circ} \mathrm{C}$. These temperature gradients are driven by heat exchange with the environment; internal heat sources are a minor contribution to the total heat flow. Under extreme conditions at the MMT, we find that these gradients and offsets double. Epps \& Fabricant (2002) have shown that these temperature gradients and temperature offsets in the optics have a negligible effect on image quality and image scale, even under extreme conditions.

If the spectrograph is exposed to an environment at a $10^{\circ} \mathrm{C}$ different temperature in the process of changing aperture masks or filters, we find the optics will experience less than $0.1^{\circ} \mathrm{C}$ temperature gradients, comparable to those experienced in normal operation without opening the spectrograph. The thermal stresses associated with these gradients are negligible.

Our calculations show that the optical bench will experience a peak $0.1^{\circ} \mathrm{C}$ axial gradient in normal operation from external temperature changes and an additional $0.1^{\circ} \mathrm{C}$ axial gradient when the spectrograph is opened to change filters. The time constants for these gradients are 12 and $4 \mathrm{hr}$, respectively. The resulting time-dependent deformations of the optical bench will tilt the grating mount and cause 0.05 and $0.15 \mathrm{pixel} \mathrm{hr}^{-1}$ image drifts at the detector, respectively. Overall, we conclude that the current Binospec design has acceptable thermal properties.

Further thermal modeling will be helpful as we proceed to the detailed design of Binospec. Thermally isolating the optical bench from the telescope mounting flange will reduce the conductive heat flow into the spectrograph and reduce the temperature gradients in the optical bench. Providing (passive) ventilation around the optical bench will allow convection to equilibrate the temperature of the spectrograph interior. Mounting motors on thermal standoffs will prevent local hot spots in the structure and allow radiation and convection to distribute heat around the spectrograph. Radiation shields on the motors may also be helpful to prevent motors from directly affecting sensitive optical surfaces. The spectrograph electronics will be mounted in a temperature-controlled enclosure that is insulated from the spectrograph interior.

We thank Bob Fata and Jack Barberis for their important contributions to this project and Henry Bergner for providing the finite-element model of the optical bench. We thank Brian Sutin for pointing out that the lens-coupling fluid may significantly affect temperature gradients and time constants in the lens groups. Steve West kindly supplied the MMT dome temperature data.

\section{REFERENCES}

Epps, H. W. 1998, Proc. SPIE, 3355, 111

Epps, H. W., \& Fabricant, D. G. 2002, PASP, 114, 1252

Fabricant, D. G., Fata, R. G., \& Epps, H. W. 1998, Proc. SPIE, 3355, 232

Fata, R. G., \& Fabricant, D. G. 1993, Proc. SPIE, 1998, 32 1998, Proc. SPIE, 3355, 275
Incropera, F. P., \& Dewitt, D. P. 1996, Fundamentals of Heat Transfer (4th ed.; New York: Wiley)

Milone, A. A. E., Heller, C., \& McAfee, J. 1999, MMT Technical Memorandum 99-1 (Mount Hopkins: MMT)

Roark, R. J., \& Young, W. C. 1989, Roark's Formulas for Stress and Strain (6th ed.; New York: McGraw-Hill) 